\title{
Estudo comparativo de anastomoses colorretais com anel biofragmentável e com grampeador em cães submetidos à irradiação gama pré-operatória ${ }^{1}$
}

\author{
José Mauro dos Santos \\ Delcio Matos $^{3}$ \\ Luís César Fernandes ${ }^{4}$ \\ João Péricles da Silva Junior ${ }^{5}$ \\ Marisa Helena da Silva ${ }^{6}$ \\ Fabrício Duarte ${ }^{7}$
}

Santos JM, Matos D, Fernandes LC, Silva Junior JP, Silva MH, Duarte F. Estudo comparativo de anastomoses colorretais com anel biofragmentável e com grampeador em cães submetidos à irradiação gama pré-operatória. Acta Cir Bras [serial online] 2003 Nov-Dez;18(6). Disponível em URL: http://www.scielo.br/acb.

RESUMO - Objetivo: Comparar os resultados precoces entre anastomoses colorretais realizadas com anel biofragmentável e com grampeador circular após irradiação pélvica. Métodos: Foram utilizados 40 cães beagle, em dois grupos de 20 animais, submetidos ou não à irradiação gama pré-operatória, com dose única de 500 cGy. Cada grupo foi dividido em dois subgrupos de dez animais, sendo denominado A o grupo em que se utilizou o anel biofragmentável e B aquele em que se empregou o grampeador. Os animais foram submetidos à secção completa do intestino grosso ao nível da junção retossigmóide, com reconstrução do trânsito sendo efetuada por uma das técnicas, após sorteio. Os animais foram sacrificados no sétimo dia de período pós-operatório. Resultados: Houve dois óbitos antes da reoperação: um em animal do grupo teste, por ruptura da anastomose realizada com anel biofragmentável; outro por peritonite generalizada em animal do grupo controle com anastomose efetuada por grampeador. Aderências e infecções de parede ocorreram de forma similar. A análise histológica convencional e a análise morfométrica realizada por computador das áreas de cicatrização anastomótica identificaram comportamento análogo entre os grupos e subgrupos estudados. Conclusão: Os resultados precoces das técnicas empregadas para anastomose intestinal foram semelhantes nas condições de risco estudadas.

DESCRITORES - Cirurgia de cólon e reto. Anastomose cirúrgica. Radioterapia. Cães.

\section{Introdução}

O intuito de obtenção de melhor cicatrização intestinal, com construção de suturas intestinais de modo mais fácil e padronizado, com menor índice de deiscências, tem levado ao estudo de métodos alternativos de anastomoses.

Steichen e Ravitch ${ }^{1}$ Fraser ${ }^{2}$ referem que o primeiro grampeador idealizado para a realização de suturas digestivas foi desenvolvido por Humer Hültl, em 1908. A partir de então tais aparelhos tiveram grande desenvolvimento, com aperfeiçoamento na extinta União Soviética nas décadas de 50 e $60^{3,4}$. Atualmente os grampeadores foram incorporados à práti-

1 Trabalho realizado no Laboratório de Técnica Operatória e Cirurgia Experimental da Universidade Federal de Santa Catarina (UFSC) e pela Disciplina de Técnica Operatória e Cirurgia Experimental da Universidade Federal de São Paulo - Escola Paulista de Medicina (UNIFESP - EPM). 2 Professor Adjunto - Doutor da Disciplina de Proctologia da Universidade Federal de Santa Catarina.

3 Professor Adjunto, Livre-Docente e Coordenador do Programa de Pós-Graduação em Gastroenterologia Cirúrgica da Universidade Federal de São Paulo - Escola Paulista de Medicina.

4 Cirurgião Geral, Mestre e Doutor pelo Programa de Pós-Graduação em Gastroenterologia Cirúrgica, Universidade Federal de São Paulo - Escola Paulista de Medicina.

5 Professor Adjunto - Doutor do Departamento de Anatomia Patológica da Universidade Federal de Santa Catarina.

6 Médica Residente de Cirurgia Geral do Hospital Universitário da UFSC.

7 Médico Residente de Cirurgia Cardiovascular do Hospital Georges Pompidou - Paris - França. 
ca cirúrgica, em intervenções convencionais e videolaparoscópicas.

A idéia de realizar suturas intestinais homogêneas e com reações teciduais mínimas levou à pesquisa de novos métodos de anastomose intestinal, sem permanência de materiais de sutura estranhos ao organismo nas áreas de cicatrização; surgiram, então, anastomoses por compressão ou sem sutura.

As primeiras anastomoses por compressão foram realizadas experimentalmente por Henroz e por Denans, em $1826^{5,6}$. Em 1892, Murphy ${ }^{7}$ efetuou estudos experimentais e clínicos utilizando anéis metálicos que se acoplavam e originavam anastomoses com inversão de paredes intestinais e aposição sero-serosa, com necrose parcial da parede digestiva e destacamento com eliminação pela evacuação. Esses anéis tiveram grande aceitação, sendo conhecidos como botões de Murphy, posteriormente abandonados inclusive por provocarem quadros obstrutivos nas anastomoses.

Em 1985, Hardy Jr. e col. ${ }^{8}$ desenvolveram o anel anastomótico bioframentável (Valtrac (®), composto de ácido poliglicólico e sulfato de bário. $\mathrm{O}$ anel, com duas partes acopláveis, fragmentava-se e era eliminado nas fezes em 2 a 4 semanas de período pós-operatório. Em 1987 estudos clínicos confirmaram os bons resultados experimentais 9 . Diversos estudos foram desenvolvidos a partir de então $0^{9-17}$

As anastomoses cólicas e colorretais são as mais sujeitas às deiscências no trato digestivo ${ }^{18}$. São diversos os fatores de risco possíveis: tensão na linha de sutura, fluxo sanguíneo local inadequado, infecção, obstrução intestinal, trauma, uso de drenos rígidos, desnutrição, efeito de corticoesteróides e irradiação prévia.

Estudos experimentais ${ }^{19-21}$ e clíni$\cos ^{22}$ têm sugerido a utilização da irradiação com 500 cGy no período pré-operatório no tratamento de linfossarcoma de Gardner e de adenocarcinoma de reto, para inativação de células tumorais, com complementação no período pós-operatório.

Em 1978, Mohiuddin e Kramer ${ }^{23}$ apresentaram proposta de radioterapia combinada pré e pós-operatória para o tratamento dos adenocarcinomas retais. Por essa proposta, dever-se-ia utilizar uma dose única de irradiação gama de 500 cGy até 24 horas antes do ato cirúrgico. Após a cirurgia, em intervenções radicais, portadores de tumores que ultrapassassem os limites da parede intestinal ou que apresentassem comprometimento ganglionar seriam encaminhados para radioterapia complementar com 4500 cGy, em 25 frações de 180 cGy, a partir da terceira semana pós-operatória. Utilizando esse esquema de tratamento alguns autores relataram um aumento da sobrevida para pacientes com neoplasia retal ${ }^{24,25}$.

Pela freqüência da utilização de radioterapia pélvica no período pré-operatório na prática clínica, seria interessante verificar experimentalmente se anastomoses por compressão com anel biofragmentável, nessa condição de risco, permitiriam a obtenção de resultados cirúrgicos melhores do que métodos tradicionalmente utilizados, como a sutura mecânica com grampos metálicos. Assim resolveu-se analisar experimentalmente anastomoses com anel biofragmentável sob risco de cicatrização, representado pela irradiação pélvica pré-operatória.

\section{Métodos}

Este projeto de pesquisa teve início após análise e aprovação pela Comissão de Ética da Universidade Federal de São Paulo - Escola Paulista de Medicina (UNIFESP-EPM). Foi efetuado no Laboratório de Técnica Operatória e Cirurgia Experimental do Departamento de Clínica Cirúrgica da Universidade Federal de Santa Catarina (UFSC), Florianópolis $\mathrm{SC}$; as intervenções foram realizadas sempre pela mesma equipe.

Foram utilizados 40 cães adultos da raça Beagle, do Biotério Central da Universidade Federal de Santa Catarina (UFSC), com peso variando de 9,0 a 19,0 kg (média de $12,2 \mathrm{~kg}$ ); idade estendendo-se de 200 a 1.829 dias (média de 601 dias).

Os animais foram distribuídos aleatoriamente em 2 grupos de 20 cães:
Grupo I (controle): animais submetidos à anastomose colorretal sem irradiação prévia;

Grupo II (teste): animais submetidos à anastomose colorretal com irradiação prévia.

Os grupos foram divididos em dois subgrupos de 10 animais, sendo denominados $\mathrm{A}$ os submetidos à anastomose colorretal por compressão com anel biofragmentável (Valtrac @) e B aqueles em que o procedimento foi efetuado por grampeador.

Os pesos e amostras sanguíneas para análise laboratorial foram obtidos antes e após o preparo intestinal efetuado. No dia anterior à cirurgia os animais foram mantidos em dieta líquida e submetidos à limpeza intestinal mecânica pela administração por via retal de 130 mililitros $(\mathrm{ml})$ de solução aquosa composta de fosfato monobásico de sódio $(16 \mathrm{~g} / 100 \mathrm{ml})$ e fosfato dibásico de sódio (6 g/100 ml) (10). No dia da intervenção os animais foram mantidos em jejum.

A anestesia foi efetuada com xilazina na dose de 3,0 mg/kg de peso corpóreo, via intramuscular, com posterior administração de pentobarbital sódico, via endovenosa, 30mg/kg de peso; houve instalação de solução fisisiológica a 0,9\%, mantida durante todo o ato operatório em infusão endovenosa contínua de $10 \mathrm{ml} / \mathrm{kg}$ por hora. Ao início do ato cirúrgico foi realizada antibioticoprofilaxia com cefoxitina sódica em dose única de 500 mg, por via endovenosa.

Os cães do grupo teste, 24 horas antes do ato operatório, foram submetidos à anestesia conforme técnica descrita e transportados até o Serviço de Radioterapia do Hospital de Caridade de Florianópolis-SC, onde foram imobilizados em decúbito dorsal e submetidos à irradiação gama por bomba de cobalto 60 . O campo de irradiação teve 10 centímetros $(\mathrm{cm})$ de diâmetro, interessando a região pélvica ${ }^{26}$, com dose única de 500 cGy, distância focosuperfície de $60 \mathrm{~cm}$, com administração por meio de portal único antero-posterior.

Para avaliação da dose de irradiação que efetivamente atingiu o reto foram utilizados dosímetros termoluminescentes 
(TLDs) de fluoreto de lítio, submetidos a leituras no Laboratório de Dosimetria do Departamento de Energia Nuclear da Universidade Federal de Pernambuco (UPFE), em Recife-PE. Três dosímetros encapsulados em cateter plástico com espessura de $0,1 \mathrm{~mm}$ foram colocados por via retal de 9 a $13 \mathrm{~cm}$ da margem anal, local a ser irradiado. A média das leituras dos dosímetros foi considerada como a dose empregada.

Os cães pertencentes ao grupo controle foram transportados, como os integrantes do grupo teste, não sendo, porém, anestesiados ou submetidos à irradiação.

Ao iniciar o ato cirúrgico, a técnica operatória a ser empregada para reconstrução do trânsito intestinal era escolhida por sorteio. Realizou-se laparotomia longitudinal mediana com extensão de $10 \mathrm{~cm}$ a partir do púbis. Foi efetuada a secção circular do cólon a aproximadamente $8 \mathrm{~cm}$ da reflexão peritoneal. Com o auxílio de pinça desenvolvida pela Davis-GeckMedical Device Division foi confeccionada sutura em bolsa na extremidade de cada segmento intestinal, com fio monofilamentar de náilon 00, utilizando-se agulha cilíndrica reta, atraumática, de $60 \mathrm{~mm}$.

As anastomoses com anéis biofragmentáveis foram efetuadas segundo método descrito por Hardy e col. ${ }^{8}$; compostos de ácido poliglicólico $(87,5 \%)$ e sulfato de bário (12,5\%), de diâmetro externo de 28 ou $31 \mathrm{~mm}$, com abertura de 1,5 e 2,0 mm entre os componentes após fechamento, fabricados pela Davis-Geck-Medical Device Division. O tamanho do anel utilizado foi adequado ao diâmetro intestinal. O dispositivo foi introduzido nos segmentos do cólon proximal e distal; as suturas em bolsa previamente efetuadas foram fechadas sobre o mesmo. A seguir foram comprimidos os componentes externos do anel, no sentido craniocaudal da alça, promovendo o fechamento do dispositivo e realizando, assim, a compressão dos segmentos interpostos com obtenção de anastomose colorretal com aposição seroserosa.

As anastomoses efetuadas com grampeador foram efetuadas de modo semelhante ao descrito por Fain e col. ${ }^{27}$, com utilização dos proximate ILS intraluminal stapler, descartáveis, com duas fileiras circulares, paralelas e alternadas de grampos de aço, de 21, 25, 29 e $33 \mathrm{~mm}$, fabricados pela Johnson \& Johnson Company e distribuídos pela Ethicon no Brasil. O diâmetro do grampeador foi adequado às dimensões da luz intestinal. Após a introdução do grampeador através do ânus realizou-se a fixação dos segmentos intestinais seccionados proximal e distal, respectivamente, à ogiva e ao corpo do aparelho, através do fechamento da sutura em bolsa. A seguir efetuou-se o grampeamento e a anastomose.

No período pós-operatório os animais foram mantidos em canis individuais e tiveram livre acesso à água e ração a partir do primeiro dia após a cirurgia. Foram avaliados diariamente, com verificação de movimentação, interatividade com o ambiente, aceitação de dieta, presença e aspecto das evacuações, eliminação do anel biofragmentável e integridade do mesmo, aspecto da ferida abdominal, presença de secreção, sinais flogísticos, deiscência de pele ou da parede abdominal.

Todos os animais foram submetidos a novo ato cirúrgico no sétimo dia de pósoperatório, com verificação de seus pesos e coleta de amostras sanguíneas para análises laboratoriais. Após serem anestesiados pela técnica previamente descrita realizou-se laparotomia mediana sobre a incisão anterior. Foi anotada a presença de secreções, abscessos e de quaisquer outras anormalidades existentes na parede abdominal. Na cavidade abdominal exposta, registrou-se em ficha padronizada de coleta de dados a presença de secreções, aderências, abscessos localizados ou peritonite generalizada. A seguir, procedeuse análise da anastomose colorretal. Foram verificados: presença de aderências, órgãos envolvidos, extensão do processo aderencial (quantificada em quadrantes), ocorrência de deiscências anastomóticas com fístulas bloqueadas ou livres, presença de dilatações da alça intestinal aferente, estenoses ou obstruções. Efetuou-se excisão em bloco da anastomose, junta- mente com os órgãos abdominais porventura aderidos.

Os animais foram sacrificados pela administração endovenosa de $10 \mathrm{ml}$ de solução de cloreto de potássio a 19,1\%. As peças cirúrgicas retiradas foram lavadas em água corrente e afixadas em lâminas de madeira para posterior análise microscópica da zona de cicatrização.

Os animais que foram a óbito no decorrer do experimento, antes do sétimo dia de pós-operatório, foram necropsiados.

A avaliação histológica convencional foi realizada no Serviço de Anatomia Patológica do Hospital Universitário da Universidade Federal de Santa Catarina. Foram seccionados quatro fragmentos longitudinais da zona anastomótica, um de cada quadrante: anterior, posterior, lateral direito e lateral esquerdo. Em caso de deiscência, os cortes foram do tecido adjacente à mesma. $\mathrm{O}$ material foi processado e incluído em parafina, de onde foram obtidos cortes histológicos com quatro micra, que foram corados pelo método de hematoxilina-eosina (H.E. ${ }^{28}$. Os parâmetros foram estudados de modo semelhante ao efetuado por Fernandes ${ }^{29}$, com modificações. Foram analisados em relação à presença e intensidade na região cicatricial: processo inflamatório, fibrose, granulomas de corpo estranho, desalinhamento das camadas teciduais na anastomose, ulceração de mucosa, esporão de mucosa e peritonite fibrinosa.

As lâminas utilizadas no estudo histológico convencional foram também avaliadas por método de digitalização de imagens. As imagens obtidas das anastomoses com anel biofragmentável e com grampeador foram digitalizadas e analisadas pelo programa DIRACOM- $3^{12,30}$. Por meio de subtração de imagens foi possível realizar análise quantitativa da reação inflamatória tecidual existente no processo de cicatrização, por cálculo da presença relativa percentual de núcleos celulares, matriz protéica e espaço intersticial. Para realização desse cálculo, foram digitalizados cinco campos escolhidos aleatoriamente entre as camadas mucosa e serosa, 
na zona anastomótica, utilizando-se a objetiva 40X do microscópio. Os três componentes da imagem foram separados, usando-se a distribuição de cor como parâmetro discriminante. Na etapa seguinte calculouse a área ocupada pelos componentes em cada um dos cinco campos da lâmina e utilizou-se a média como valor final. Também foram medidas, linearmente, as espessuras das camadas submucosas do intestino adjacentes à área cicatricial e a espessura da cicatriz na área da anastomose.

A análise estatística foi realizada na disciplina de Bioestatística do Departamento de Medicina Preventiva da UnifespEpm. Na comparação das variáveis, na véspera da cirurgia, no dia do ato cirúrgico e no sétimo dia de pós-operatório, dentro de cada subgrupo, foi empregada a análise de variância por postos de Friedman ${ }^{31}$, complementada pelo teste de comparações múltiplas, quando houve significância estatística ${ }^{32}$. O teste de MannWhitney $^{31}$ foi usado para comparar as variáveis estudadas entre os subgrupos A e B e os grupos controle e teste, em cada tempo do experimento. Empregouse o teste exato de Fisher ${ }^{31}$ para comparar a presença ou ausência de peritonite fibrinosa e granuloma de corpo estranho entre os grupos e subgrupos estudados. Fixouse em 0,05 ou $5 \%$ o nível de rejeição da hipótese de nulidade, assinalando-se os valores significantes.

\section{Resultados}

Foi elevada a incidência de infecção de parede abdominal nos animais operados, tendo ocorrido em 12 cães do grupo I (60\%), sendo 4 do grupo IA e 8 do grupo IB; no grupo II, 16 animais tiveram infecção da ferida operatória (80\%), sendo 8 do grupo IIA e 8 do grupo IIB. Ocorreram cinco eviscerações pós-operatórias, sendo 2 (20\%), em animais do grupo IB, 2 (20\%) em animais do grupo IIA e $1(10 \%)$ em animal do grupo IIB; todos os animais foram reoperados à exceção de um animal do grupo IB, com evisceração detectada no dia da reoperação. Não houve diferença estatisticamente significante entre os grupos (Tabela 1).
$\mathrm{Na}$ avaliação macroscópica das anastomoses, verificou-se que a aderência das mesmas às estruturas vizinhas ocorreu em praticamente todos os animais operados, sem diferença entre os grupos; deiscência parcial da anastomose (bloqueada e sem peritonite), ocorreu em 3 animais, sendo 1 do grupo IA e 2 do grupo IIA, mas sem repercussão clínica; em 1 animal do grupo IA houve migração do anel biofragmentável com obstrução da luz intestinal e conseqüente rotura da anastomose e óbito no sexto dia de pósoperatório; em um animal do grupo IIB ocorreu peritonite difusa sem rotura da anastomose e óbito no terceiro dia de pósoperatório(Tabela 2).

Em três (30\%) animais do grupo IA (controle) e um (10\%) do grupo IIA (teste), o anel biofragmentável foi eliminado nas fezes sem fragmentação; em dois (20\%) animais do grupo IA o anel foi encontrado livre na luz intestinal, sendo um íntegro e outro fragmentado. Em cinco (50\%) animais do grupo IA e em 9 (90\%) do grupo IIA o anel foi encontrado fixo e íntegro na zona de anastomose.

TABELA 1 - Complicações da parede abdominal no período pós-operatório nos cães dos grupos controle (I) e teste (II), com anastomoses por anel biofragmentável (A) e por grampeador $(\mathrm{B})$.

\begin{tabular}{lcccccccc}
\hline & \multicolumn{3}{c}{ Grupo Controle (I) } & \multicolumn{3}{c}{ Grupo Teste (II) } \\
\cline { 2 - 10 } Complicações & \multicolumn{2}{c}{ Anel } & \multicolumn{2}{c}{ Grampeador } & \multicolumn{2}{c}{ Anel } & \multicolumn{2}{c}{ Grampeador } \\
\cline { 2 - 10 } & \multicolumn{2}{c}{ biofragmentável(A) } & (B) & biofragmentável (A) & (B) \\
\hline \multirow{2}{*}{ Infecção } & $\mathrm{f}$ & $\%$ & $\mathrm{f}$ & $\%$ & $\mathrm{f}$ & $\%$ & $\mathrm{f}$ & $\%$ \\
Sangramento & 4 & 40 & 8 & 80 & 8 & 80 & 8 & 80 \\
Deiscência cutânea & 3 & 30 & 6 & 60 & 2 & 20 & 3 & 30 \\
Evisceração & 0 & 0 & 2 & 20 & 2 & 20 & 1 & 10 \\
\hline
\end{tabular}

TABELA 2 - Avaliação macroscópica das anastomoses intestinais com anel biofragmentável (A) e com grampeador (B), dos grupos controle (I) e teste (II), no $7^{\circ}$ dia de pós-operatório.

\begin{tabular}{|c|c|c|c|c|c|c|c|c|}
\hline \multirow{3}{*}{$\begin{array}{l}\text { Achado } \\
\text { operatório }\end{array}$} & \multicolumn{4}{|c|}{ Anel biofragmentável (A) } & \multicolumn{4}{|c|}{ Grampeador (B) } \\
\hline & \multicolumn{2}{|c|}{$\begin{array}{c}\text { Grupo } \\
\text { controle (I) }\end{array}$} & \multicolumn{2}{|c|}{$\begin{array}{c}\text { Grupo } \\
\text { teste (II) }\end{array}$} & \multicolumn{2}{|c|}{$\begin{array}{c}\text { Grupo } \\
\text {-controle (I) }\end{array}$} & \multicolumn{2}{|c|}{$\begin{array}{c}\text { Grupo } \\
\text { teste (II) }\end{array}$} \\
\hline & $\mathrm{f}$ & $\%$ & $\mathrm{f}$ & $\%$ & $\mathrm{f}$ & $\%$ & $\mathrm{f}$ & $\%$ \\
\hline Aderência & 10 & 100 & 10 & 100 & 10 & 100 & 10 & 100 \\
\hline $\begin{array}{l}\text { Deiscência parcial } \\
\text { da anastomose (bloqueada) }\end{array}$ & 1 & 10 & 2 & 20 & 0 & 0 & 0 & 0 \\
\hline $\begin{array}{l}\text { Deiscência da anastomose } \\
\text { (peritonite e óbito) }\end{array}$ & 1 & 10 & 0 & 0 & 0 & 0 & 0 & 0 \\
\hline $\begin{array}{l}\text { Peritonite difusa e óbito } \\
\text { (sem deiscência) }\end{array}$ & 0 & 0 & 0 & 0 & 0 & 0 & 1 & 10 \\
\hline $\begin{array}{l}\text { Obstrução parcial } \\
\text { da anastomose } \\
\text { (dilatação aferente) }\end{array}$ & 2 & 20 & 1 & 10 & 0 & 0 & 0 & 0 \\
\hline
\end{tabular}


TABELA 3 - Incidência de desalinhamento das camadas teciduais nas anastomoses com anel biofragmentável (A) e com grampeador (B), nos grupos controle (I) e teste (II), no $7^{\circ}$ dia de pós-operatório.

\begin{tabular}{|c|c|c|c|c|c|c|c|}
\hline \multirow{3}{*}{$\begin{array}{l}\text { Desalinha- } \\
\text { mento de } \\
\text { camadas } \\
\text { I }\end{array}$} & \multicolumn{3}{|c|}{$\begin{array}{l}\text { Anel biofragmentável } \\
\text { (A) }\end{array}$} & \multicolumn{3}{|c|}{$\begin{array}{l}\text { Grampeador (B) } \\
\qquad \mathrm{AxB}\end{array}$} & \\
\hline & presente & $\begin{array}{c}\text { ausente } \\
(\%)\end{array}$ & presença & $\begin{array}{c}\text { presente } \\
(\%)\end{array}$ & ausente & presença & \\
\hline & 8 & 2 & 80,0 & 2 & 8 & 20,0 & $p=0,0045$ \\
\hline II & 9 & 1 & 90,0 & 3 & 7 & 30,0 & $\begin{array}{r}A>B \\
\mathbf{p}=\mathbf{0 , 0 1 7 4}\end{array}$ \\
\hline & & & & & & & $A>B$ \\
\hline
\end{tabular}

A avaliação histológica convencional das anastomoses mostrou que o processo inflamatório crônico e a fibrose ocorreram em todos os animais operados. Granuloma de corpo estranho ocorreu em $20 \%$ dos animais do grupo IA e em nenhum do grupo IIA, em $20 \%$ dos animais do grupo IB e $40 \%$ no grupo IIB, sem diferença estatisticamente significante.

O desalinhamento das camadas teciduais ocorreu em $8(80 \%)$ das anastomoses do grupo IA, 9 (90\%) do grupo IIA, 2 (20\%) do grupo IB e 3 (30\%) do grupo IIB, o que demonstrou que em anastomoses com grampeador se obteve melhor alinhamento que nas anastomoses com anel, com significância estatística (Tabela 3).

O esporão de mucosa para a luz intestinal ocorreu em 4 (40\%) dos animais do grupo IA, 6 (60\%) do grupo IIA, 3 (30\%) do grupo IB e 1 (10\%) do grupo IIB, ou seja, ocorreu com maior frequiência nos animais em que se empregou o anel biofragmentável, com significância estatística nos animais irradiados $(\mathrm{p}=0,0399)$.

Ulceração mucosa foi constante em todas as anastomoses e a sua medida mostrou que a mesma foi discretamente maior quando se empregou o grampeador, mas essa diferença não foi estatisticamente significante e não sofreu influência da irradiação. A peritonite fibrinosa ocorreu entre 70 a $80 \%$ das anastomoses sem diferença estatisticamente significante entre os grupos estudados.
A análise computadorizada das anastomoses estudadas demonstrou que as áreas correspondentes aos núcleos celulares, à matriz protéica tecidual e ao líquido intersticial (edema) foram semelhantes nos quatro grupos estudados, sem diferença estatística.

A espessura em mm da submucosa adjacente às anastomoses intestinais foi em média de 0,91 mm no grupo IA, 0,61 $\mathrm{mm}$ no grupo IIA, $0,79 \mathrm{~mm}$ no grupo IB e 0, $74 \mathrm{~mm}$ no grupo IIB, diferença com significância estatística nos animais irradiados.

A medida da espessura em $\mathrm{mm}$ do tecido cicatricial das anastomoses mostrou, em média, 5,79 $\mathrm{mm}$ nos animais do grupo IA; 5,03 mm nos animais do grupo IB; 7,29 $\mathrm{mm}$ nos animais do grupo IIA e 3,39 mm nos animais do grupo IIB; esses resultados mostraram que as anastomoses realizadas com o grampeador são mais delgadas que as realizadas com o anel biofragmentável e essa diferença foi estatisticamente significante nos animais irradiados.

\section{Discussão}

O uso nesta pesquisa de cães da raça Beagle, oriundos do biotério central da UFSC, de pesos e idades conhecidos, criados exclusivamente para experimentação, caracterizou a homogeneidade da amostra utilizada. Também o fato da mesma equipe de cirurgiões realizar todos os atos cirúrgicos, bem como a padronização prévia das técnicas anastomóticas, anestésicas e do fator de risco estudado resultou em procedimentos bastante homogêneos, permitindo a sua comparação.

A frequência de infecções de parede abdominal em trabalhos experimentais em cães irradiados previamente é bastante variável, oscilando de $0^{33}$ a $38 \%{ }^{34}$. As hérnias da parede abdominal ocorrem em torno de $30 \%{ }^{35}$. No presente experimento houve grande número de complicações pós-operatórias relacionadas à parede abdominal, com percentuais maiores nos animais submetidos à irradiação prévia e naqueles em que se utilizou o grampeador como método de anastomose intestinal, mas ambos sem significância estatística. Talvez preparo intestinal pré-operatório mecânico insuficiente, apenas com um enema, com manuseio de alças em geral com conteúdo fecal, possa ser responsabilizado pelo resultado encontrado.

$\mathrm{Na}$ literatura, é referido um índice mais alto de aderências pós-operatórias nas anastomoses cólicas em que se utiliza o anel biofragmentável em comparação com sutura manual em cães ${ }^{12}$ ou com grampeador $^{36}$. o entanto, neste experimento, todos os animais apresentaram aderências perianastomóticas, independentemente da técnica utilizada e sem influência da irradiação pré-operatória.

A semelhança no resultado das anastomoses do intestino grosso realizadas com anel biofragmentável, com grampeador e com sutura manual tem sido evidenciada em estudos experimentais $^{11,13,37}$ e clínicos ${ }^{13,38-40}$. Existem algumas vantagens do anel biofragmentável em relação aos outros métodos, como ausência de corpos estranhos nas anastomoses, possibilidade de utilização em qualquer ponto do intestino dentro da cavidade abdominal e representação de técnica padronizada $^{40}$.

A multiplicidade de esquemas de tratamento radioterápico adjuvante préoperatório para os adenocarcinomas retais, na prática clínica, tem motivado diversos estudos experimentais. Smith et al. ${ }^{33}$, utilizando irradiação fracionada pré- 
operatória com 5000 cGy em cães, relataram quatro deiscências em dez anastomoses colorretais realizadas com anel biofragmentável com abertura de $1,5 \mathrm{~mm}$ entre os dispositivos, que resultaram em três óbitos. Referiram uma deiscência em dez suturas manuais e nenhuma nas dez anastomoses com grampeador e com anel com abertura de 2,0 mm. Esses autores referiram ser de importância fundamental o tamanho do espaço entre os dois dispositivos do anel após o seu acoplamento, pois o de abertura menor causaria mais necrose e, conseqüentemente, maior facilidade de ocorrência de fístulas. Croston et al. ${ }^{34}$ relataram um índice de deiscências de $23 \%$ nas anastomoses colorretais com anel bioframentável realizadas após irradiação fracionada com 6000 cGy, em cães, enquanto que, em estudo anterior, tiveram um índice de deiscência de $70 \%$ e $80 \%$, respectivamente, quando se utilizou a sutura manual e o grampeador. Concluíram então que, com essa dose de irradiação, as anastomoses colorretais manuais e com grampeador são proibitivas e que o anel biofragmentável poderia representar uma alternativa aparentemente mais segura para a restauração do trânsito intestinal.

No presente experimento ocorreram quatro deiscências nos vinte cães submetidos a anastomoses com anel biofragmentável. Dessas, três foram parciais, posteriores, bloqueadas e sem repercussão clínica, sendo uma em animal do grupo controle e duas nos animais submetidos a radioterapia prévia. Um animal do grupo controle apresentou migração do anel bioframentável, obstrução intestinal distal à anastomose, ruptura da mesma, peritonite e óbito no sexto dia de pós-operatório. Nos animais em que as anastomoses foram realizadas com grampeador ocorreu um óbito, em espécime do grupo controle, no terceiro dia de pósoperatório, por peritonite purulenta generalizada. A necrópsia desse animal mostrou anastomose íntegra. A causa da infecção não foi esclarecida, podendo ter sido provocada por contaminação durante a introdução e manuseio do grampeador em intestino com preparo inadequado. As complicações relacionadas às anastomoses ocorreram nos grupos controle e teste com maior tendência a fistulização nos animais com anastomose por compressão, mas sem significância estatística.

A eliminação do anel biofragmentável nas fezes tem ocorrido, segundo estudos, entre a segunda e terceira semana de pós-operatório ${ }^{8}$, não tendo sido relatada influência da irradiação prévia nesse fato $^{33,34,37}$. Neste experimento houve a eliminação do anel de forma íntegra em quatro animais, sendo três do grupo controle e um do grupo teste, aparentemente sem influência da irradiação pré-operatória. Outros autores, em estudos em cães, também relataram a eliminação do ane íntegro nas fezes no pós-operatório ${ }^{12,19}$.

Em dois animais do presente estudo o anel foi encontrado livre na luz intestinal; um no sexto dia de pós-operatório, íntegro, que causou obstrução intestinal e óbito. Os demais animais apresentavam o anel fixo à anastomose na reoperação. A eliminação precoce de alguns anéis não influenciou na cicatrização das anastomoses, sendo a evolução desses animais semelhante à dos demais; no entanto, o fato de se encontrarem anéis livres e íntegros na luz intestinal faz supor que os mesmos possam ter sido eliminados por insuficiência de pressão de fechamento ou por necrose tecidual ocorrida antes da sua fragmentação, o que é preocupante, pois o deslocamento precoce dos anéis da zona anastomótica leva teoricamente à perda do material de sustentação das anastomoses. Isso, aliado ao risco de progressão da necrose da parede intestinal, poderia levar a um aumento do risco de deiscências nesses animais.

A análise histológica convencional das anastomoses colorretais mostrou que o processo inflamatório e a fibrose foram semelhantes nas duas técnicas utilizadas. A presença de granulomas de corpo estranho ocorreu de maneira semelhante nos animais, em ambas as técnicas de anastomose, o que não deveria ocorrer, já que um dos métodos tem como premissa fundamental a não permanência de corpos estranhos na linha de sutura intestinal.
O desalinhamento das camadas teciduais foi menor nos cães em que a anastomose intestinal foi realizada com grampeador, tanto nos animais irradiados como nos do grupo controle, de maneira estatisticamente significante. Em estudos realizados anteriormente, o anel biofragmentável apresentou anastomoses mais alinhadas que as anastomoses manuais ${ }^{12,29}$, fato talvez explicado pela maior variabilidade a que está sujeito o procedimento efetuado por intervenção manual.

A ocorrência de esporões de mucosa em direção à luz intestinal foi menor nos animais submetidos à anastomose com grampeador, diferença estatisticamente significante no grupo previamente irradiado, fato talvez explicado pela secção parcial do excesso de tecido que ocorre no momento do grampeamento.

A ulceração de mucosa foi constante em todos os animais, ocorrência também relatada em outros experimentos ${ }^{8,10,11,29}$.

A avaliação histológica por digitalização de imagens foi importante por possibilitar análise quantitativa precisa e homogênea da zona cicatricial das anastomoses colorretais. A reparação tecidual em cães ocorre de maneira semelhante em ambas às técnicas e não houve problemas decorrentes da irradiação gama com 500 cGy em dose única aplicada no préoperatório.

Em estudos ${ }^{33,34}$ houve o relato de que anastomoses colorretais com anel biofragmentável, após altas doses de irradiação gama, demonstraram resultados semelhantes e complicações análogas às realizadas com grampeador. No presente estudo, utilizando dose única de irradiação gama pré-operatória, os métodos de anastomose propiciaram resultados idênticos. É necessário que se realizem avaliações tardias das anastomoses colorretais experimentais, com o anel biofragmentável após emprego de irradiação gama pré-operatória, para comparação com resultados descritos ${ }^{19,20}$.

Apesar dos resultados obtidos neste estudo experimental, o anel biofragmentável utilizado nos dias atuais parece não ser o dispositivo ideal para a realização 
das anastomoses intestinais. Seu lúmen ainda parece ser estreito, talvez necessitando de ampliação para que não ocorra obstrução da luz e impactação fecal com risco de deiscência anastomótica pelo aumento de gradiente pressórico, como ocorrido neste experimento. Modificações no dispositivo seriam úteis, principalmente para aplicação em anastomoses colorretais baixas, como desenvolvimento de dispositivo aplicador e confecção de anéis com metades destacáveis, visando facilitar sua introdução na luz intestinal, conforme sugerido por Fernandes ${ }^{29}$. Por outro lado, o manuseio do anel biofragmentável revelou-se tão simples e fácil quanto anastomoses intestinais realizadas com grampeador.

\section{Conclusão}

Este experimento pôde mostrar que, em cães, para a realização de anastomoses colorretais sob risco por irradiação gama pré-operatória com 500 cGy, a utilização de anel biofragmentável se constitui em técnica segura, de fácil realização e com complicações precoces semelhantes às ocorridas em comparação com o uso de grampeador endoluminar.

\section{Referências}

1. Steichen FM, Ravitch MM. Mechanical sutures in surgery. Br J Surg 1973; 60:191-7.

2. Fraser I. An historical perspective on mechanical aids in intestinal anastomosis. Surg Gynecol Obstet 1982; 155:566-74.

3. Amosov NM, Berezovsky KK. Pulmonary resection with mechanical suture. J Thoracic Cardiovasc Surg 1961; 41:325-35.

4. Androsov PI. New instruments for thoracic surgery. Dis Chest 1963; 44:590-7.

5. Ravitch MM. Developments of intestinal anastomotic devices. South Med J 1982; 75:1520-30.

6. Denans FN. Entérorraphie. Arch Gen Med 1826;2:618.

7. Murphy JB. Cholecysto-intestinal, gastrointestinal, entero-intestinal, anastomosis, and approximation without sutures: original research. Med Rec 1892; 42:665-76.

8. Hardy TG, Pace WG, Maney JW, Katz AR, Kaganov AL. A biofragmentable ring for sutureless bowel anastomosis: an experimental study. Dis Colon Rectum $1985 ; 28: 484-90$

9. Hardy TG, Aguilar PS, Stewart WR, Katz AR, Maney JW, Costanzo JT. Initial clinical experience with a briofragmentable ring for sutureless bowel anastomosis. Dis Colon Rectum 1987; 30:55-61.

10. Polonio B. Estudo comparativo entre as anastomoses colorretais em plano único extramucoso e com anel biofragmentável no reto extraperitonial de cães [Tese Mestrado]. Universidade Federal de São Paulo - Escola Paulista de Medicina; 1992.

11. Czeczko NG. Estudo comparativo entre as anastomoses colorretais mecânicas com grampeador e anel biofragmentável no reto extraperitonial de cães [Tese - Doutorado]. Universidade Federal do Paraná; 1992.

12. Matos D, Saad SS, Franceschi Júnior O Barreto EP, Novelli MD. Estudo experimental comparativo entre anastomose colocólica com sutura manual e com anel biofragmentável. Rev Ass Med Bras 1993; 39:201-6.

13. Gullichsen R, Ovaska J, Havia T, Yrjänä J, Ekfors T. What happens to the Valtrac ${ }^{\circledR}$ anastomosis of the colon? Dis Colon Rectum 1993; 36:362-5.

14. Habr-Gama A, Soares JH, Campos FGMC Anastomose intestinal sem sutura: resultado inicial com o anel biofragmentável (Valtrac (B). Rev Bras ColoProctol 1994; 14:225-9.

15. Polonio B, Repka J C D, Grinaldi D M, Novo N F, Juliano Y, Nigro A J T. Anastomoses esôfago-esofágicas cervicais término-terminais por invaginação e com anel biofragmentável: estudo comparativo em cães. Acta Cir Bras 1996; 11:51-7.

16. Soares JH, Nigro AJT, Lima CAP, Stávale JN, Juliano Y, Novo NF. Anastomoses esôfago-gástricas com anel biofragmentável e por invaginação: estudo experimental em cães. Acta Cir Bras 1996; 11:191-9.

17. Saad SS, Matos D. Estudo clínico comparativo entre anastomose colocólica com anel biofragmentável e com sutura manual não absorvível. Rev Col Bras Cir 2000; 27:173-80.

18. Hawley PR. Causes and prevention of colonic anastomotic breakdown. Dis Colon Rectum 1973; 16:272-7.

19. Powers WE, Tolmach LJ. Pre-operative radiation therapy: biological basis and experimental investigation. Nature 1964; 201:272-3

20. Powers WE, Palmer LA. Biologic basis of preoperative radiation treatment. Am J Roentgenol 1968; 102:176-92.

21. Nias AHW. Radiobiological aspects of preoperative irradiation. Br J Radiol 1967; 40:166-9.
22. Rider WD, Palmer JA, Mahoney LJ, Robertson CT. Preoperative irradiation in operable cancer of the rectum: report of the Toronto Trial. Can J Surg 1977; 20:335-8.

23. Mohiuddin M, Kramer S. Adjuvan radiotherapy preoperative, postoperative, or both: a proposal for a new approach Cancer Clin Trials 1978; 1:93-7.

24. Gunderson LL, Dosoretz DE, Hedberg SE, Blitzer PH, Rodkey G, Hoskins B. Lowdose preoperative irradiation, surgery, and elective postoperative radiation therapy for resectable rectum and rectosigmoid carcinoma. Cancer 1983; 52:446-51.

25. Mohiuddin M, Derdel J, Marks G, Kramer S. Results of adjuvant radiation therapy in cancer of the rectum: Thomas Jefferson University Hospital experience. Cancer 1985; 55:350-3.

26. Morgenstern L, Sanders G, Wahlstrom E, Yadegar J, Amodeo P. Effect of preoperative irradiation on healing of low colorectal anastomoses. Am J Surg 1984; 147:246-9.

27. Fain SN, Patin CS, Morgenstern L. Use of a mechanical suturing apparatus in low colorectal anastomosis. Arch Surg 1975; 110:1079-82.

28. Michalany J. Técnica histológica em anatomia patológica. São Paulo: EPU, 1980.

29. Fernandes LC. Estudo comparativo entre anastomoses intestinais com sutura manual e com anel biofragmentável, em cães sob a administração de corticosteróides [Tese Mestrado]. Universidade Federal de São Paulo - Escola Paulista de Medicina; 1997.

30. Novelli MD, Barreto E, Matos D, Saad SS, Borra RC. Aplicação do processamento de imagens por computador na quantificação das variáveis histopatológicas da reparação tecidual de anastomoses colocólicas em cães. Rev Ass Med Bras 1997; 43:277-82.

31. Siegel S; Castellani Jr NJ. Nonparametrics statistics. 2ed. New York: Mc Graw-Hill; 1988.

32. Hollander M, Wolfe DA. Nonparametric statistical methods. New York: John Wiley \& Sons; 1973.

33. Smith AD, Bubrick MP, Mestitz ST, Crouch FM, Johnston GR, Feeney DA. Evaluation of the biofragmentable anastomotic ring following preoperative irradiation to the rectossigmoid in dogs. Dis Colon Rectum 1988; 31:5-9.

34. Croston JK, Jacobs DM, Kelly PH, Feeney DA, Johnston GR, Strom RL. Experience with the biofragmentable anastomotic ring (BAR) in bowel preoperatively irradiated with 6000 rad. Dis Colon Rectum 1990; $33: 222-6$. 
35. Bubrick MP, Rolfsmeyer ES, Schauer RM, Feeney DA, Johnston GR, Strom RL. Effects of high-dose and low-dose preoperative irradiation on low anterior anastomoses in dogs. Dis Colon Rectum 1982; 161:136-43

36. Bundy CA, Zera RT, Onstad GA, Bilodeau LL, Bubrick MP. Comparative surgical and colonoscopic appearance of colon anastomoses constructed with sutures, staples, and the biofragmentable anastomotic ring. Surg Endosc 1992; 6:18-22.

37. Maney JW, Katz AR, Li LK, Pace WG Hardy TG. Biofragmentable bowel anastomosis ring: comparative efficacy studies in dogs. Surgery 1988; 103:56-62.

38. Choen FS, Eu KW. Circular staples versus the biofragmentable ring for colorectal anastomosis: a prospective randomized study. Br J Surg 1994; 81:1789-90.
39. Cahill CJ, Betzler M, Gruwez JA, Jeekel J, Patel JC, Zederfeldt B. Sutureless large bowel anastomosis: European experience with the biofragmentable anastomotic ring. Br J Surg 1989; 76:344-7.

40. Bubrick MP, Corman ML, Cahill CJ, Hardy TG, Nance FC, Shatney CH. Prospective, randomized trial of the biofragmentable anastomotic ring. Am J Surg 1991; 161:136-43.

Santos JM, Matos D, Fernandes LC, Silva Junior JP, Silva MH, Duarte F. Comparative study between colorectal anastomosis with biofragmentable anastomotic ring and with stapler after gamma irradiation. Acta Cir Bras [serial online] 2003 Nov-Dec;18(6). Available from URL: http://www.scielo.br/acb.

ABSTRACT - Purpose: To compare the early results of colorectal anastomosis with the biofragmentable ring and with stapler after preoperative gamma irradiation with a 500 cGy single dose. Methods: Forty beagle dogs were distributed in 2 groups of 20 animals submitted or not to preoperative gamma irradiation and named test(II) and control (I) groups respectively. Two subgroups of 10 animals were created: group A in which the biofragmentable ring was used and group B in which the stapler was used. The animals were submitted to full division of the large bowel at the rectosgmoid junction and the intestinal tube has been reconstructed by one of the two techniques randomly assigned. In the seventh postoperative day the animals were killed and histological specimens were collected for macro and microscopic analysis. Results: Two deaths occurred before reoperation: one in the biofragmentable anastomosis group and the other in the stapler anastomosis group, due to generalized peritonitis. Adhesions and wound infection have occurred equally in all groups. The standard histological analysis and computorized morphometric study showed no significant differences between the groups. Conclusion: The early results of the two anastomosis technique under risk conditions were alike.

KEY WORDS - Colorectal surgery. Surgical anastomosis. Radiotherapy. Dogs.

Conflito de interesse: nenhum

Correspondência:

Prof. Dr. Delcio Matos

Rua Napoleão de Barros, 610

04024-002 São Paulo - SP

Tel/Fax: (11)5084-7551

dmatos.dcir@epm.br
Data do recebimento: 28/08/2003

Data da revisão: 12/09/2003

Data da aprovação: 02/10/2003 